\title{
Design of the Smart Pneumatic Balance Crane Control System
}

\author{
Jiujun ZHEN ${ }^{1,2, a *}$, Lu DING $^{3, b}$, Xiaoyong WANG ${ }^{1, c}$ \\ ${ }^{1}$ School of Mechanical Engineering, Nanjing Vocational Institute of Industry Technology, Nanjing, \\ 210023, China \\ ${ }^{2}$ College of Mechanical and Electrical Engineering, Nanjing University of Aeronautics and \\ Astronautics, Nanjing, 210016, China \\ ${ }^{3}$ School of Mechanical Engineering, Nanjing Institute of Technology, Nanjing, 211167, China \\ azhenjiujun@126.com, ${ }^{*}$ corresponding author, ${ }^{\mathrm{b}} 1184583084 @ q q . c o m,{ }^{c}$ wangxy@niit.edu.cn
}

\section{Keywords: Smart; Pneumatic Balance Crane; Control System; MCU}

\begin{abstract}
Through the analysis of the way of Labor-saving flitting, a new smart pneumatic balance crane which combines micro-control technology with pneumatic technology is presented. Firstly, the overall design of the system is completed. Secondly, the principle of each functional module is described in detail, then the design of pneumatic system and control circuit of intelligent pneumatic balance crane is completed. Finally, the software is divided into different kinds of modules according to the function of each part, and network diagram of the software is designed. Then, the design and implementation of software are described in detail. By running the new smart pneumatic balance crane, it can be seen that the structure of the crane is simple, and crane system is convenient to transport and install. It can be widely used in cleaning, handling, cutting, and other occasions, and is of great significance to the new way to develop intelligent manufacturing.
\end{abstract}

\section{Introduction}

In the fields of mechanical assembly, sheet metal forming, machine tool processing and so on, the handling and assembly of large quality work-pieces are carried out mainly through multiplayer operation, electric hoist, balanced crane, industrial robot and other ways. Smart pneumatic balance crane is one kind of brand-new products, and more suitable for the precise positioning of the product handling and assembly occasions than the electric gourd [1] [2]. It is controlled by micro-controller, using the force balance principle skillfully, to form a non-gravity floating state for the work-piece in the air, that is, the gravity of the work-piece can be automatically balanced by the pressure of the gas control system, so that the operator without skilled jogging button operation, just a small operating force, can put the weight accurately into the operating space in any position, the maximum load can reach $880 \mathrm{Kg}$.

In the design of the smart pneumatic balance crane, to remove the ordinary pneumatic balance hanging reel structure, using a self-intellectual property rights, adaptive intelligent control system, successfully resolved the original pneumatic balance crane high price and maintenance complexity and other issues [3] [4].

\section{Design of the Entire Scheme}

The aerodynamic balance lifting structure is simplified by smart pneumatic balance crane. Only by the control box, ordinary cylinder, gas control valve, rack four parts [5]. The actuator directly uses the ordinary cylinder, and the dual functions of the electric hoist and the balanced crane can be accomplished by the combination of pneumatic and micro-controller. This design avoids the complexity of the reel structure and the higher price of the servo motor and control system, and increase the system flexibility and buffer function. When lifting and assembling height less than 1 meter, you can directly use the 1-meter stroke cylinder to lift the work-piece. If more than $1 \mathrm{~m}$, you 
can use the dynamic pulley zoom to get a smaller structure and a longer working distance. The system structure is shown in Figure1.

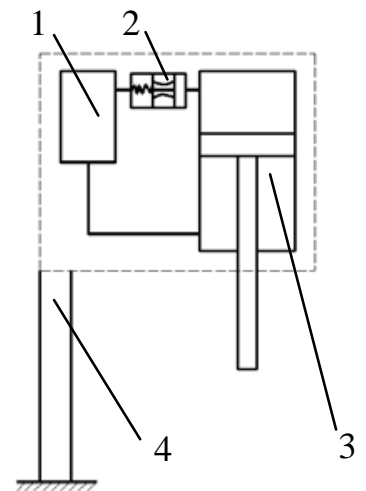

1-Control box. 2-Gas control safety valve. 3-Cylinder. 4-Rack.

Fig.1. Entire scheme

The electrical control system and the pneumatic transmission circuit are highly integrated in the control box 1. The control box is sealed and the whole system has the explosion-proof property of dangerous gas. The input and output ports of the control box include: a compressed air inlet, an exhaust port, a gas junction 1 connecting the gas control valve, a standby air inlet 2, a $24 \mathrm{~V}$ power interface, a communication interface.

The control box of pneumatic balance crane adopts 485bus which can communicate via communication interface and PC. The balance crane parameters can be set up by PC software online, including the maximum lifting weight, lifting weight adaptive balancing accuracy, etc. The mode switching switch on the control box can choose the balanced crane operation mode or the pneumatic balance crane operation mode. The air - controlled safety valve 2 can realize the pressure of the cylinder 3, which can be used to prevent lifting objects from falling when the air is suddenly broken.

\section{Design of the Hardware of Control System}

Design of the Control Plan. Control system program block diagram is shown in Figure 2.

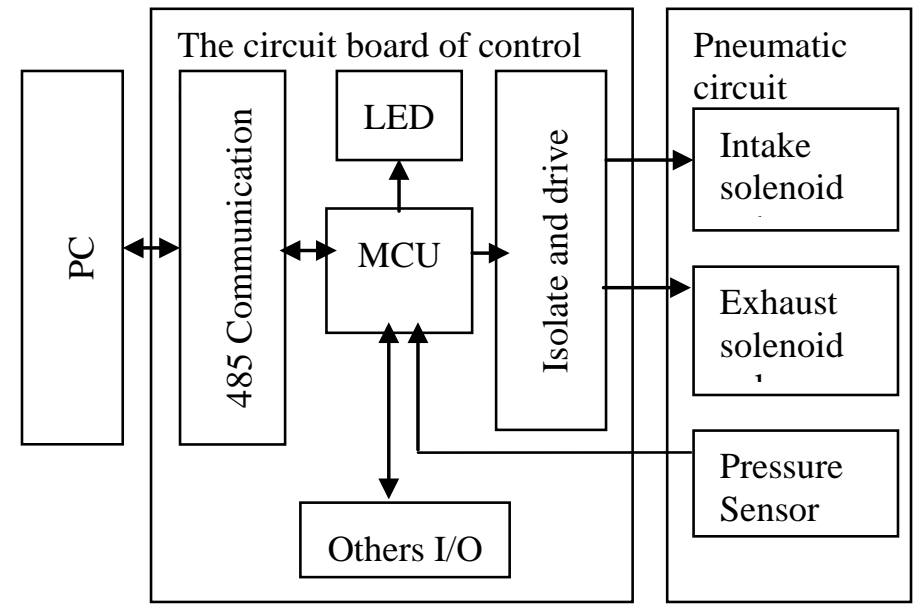

Fig.2. Control system plan diagram

The control system controls the air pressure in the cylinder by controlling the solenoid valve in the pneumatic circuit through the MCU, thus completing the control of the rise, fall and hover of the weight [6]. In order to achieve closed-loop control, the pressure sensor detects the cylinder pressure in real time and gives feedback to the MCU to determine the state of the cylinder. The pressure value is displayed by the display system module to help the operator identify the system status. The display system is designed two, one is four LED, the dynamic scan display, and the second is the upper computer software interface display. 
The input signal of the system has the analog voltage signal of the pressure sensor, the key signal, and the PC signal transmitted to the MCU.

Design of the Pneumatic Circuit. Pneumatic circuits are highly integrated in the control box, including: pressure regulator, intake solenoid valve, exhausts solenoid valve, spare solenoid valve, pressure sensor, etc. The pneumatic schematic diagram is shown in Figure 3.

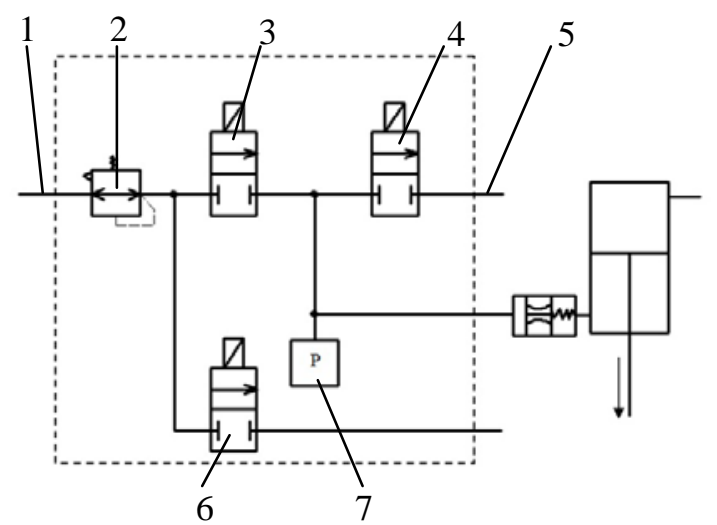

1-Air inlet. 2-Pressure regulating valve. 3-Intake solenoid valve. 4-Exhaust solenoid valve.

5-Air outlet. 6-Backup solenoid valve. 7-pressure sensor.

Fig.3. Pneumatic schematic diagram

The inlet 1 is used to provide the system with a power source (compressed air). When the intake solenoid valve 3 is energized and the exhaust solenoid valve 4 is de-energized, the compressed air passes through the gas control safety valve to the cylinder, the cylinder piston rises, lifting the weight.

When the intake solenoid valve 3 is de-energized and the exhaust solenoid valve 4 is energized, the compressed air passes through the gas control safety valve to the exhaust solenoid valve 4 . As shown in Fig2. The compressed air is discharged from the exhaust port 5 and the cylinder piston is lowered. Normally, compressed air can be passed in or out of the gas control relief valve. Ensure that the cylinder is suspended when the sudden loss of pressure is caused by the gas pipeline damage and so on because the compressed air in the cylinder cannot be exhausted through the gas control valve.

The pressure sensor $\mathrm{P}$ is used to detect the pressure change in the balance cylinder. The MCU in the control circuit controls the intake solenoid valve 3 and the exhaust solenoid valve 4 according to the feedback value of the pressure sensor to maintain the pressure balance in the cylinder.

When the pneumatic circuit is designed, the diameter of the cylinder shall be calculated first according to the lifting weight [7]. The calculation formula is shown below.

$$
F=\frac{1}{4} \cdot \pi\left(D^{2}-d^{2}\right) \cdot p \cdot \beta
$$

$F$-Thrust $(\mathrm{N})$; $D$-Cylinder bore $(\mathrm{mm})$; $d$-Piston rod diameter $(\mathrm{mm}) ; \beta$-Load rate; $p$-Supply air pressure $(\mathrm{MPa})$. The cylinder is driven by inertial load, the load will produce inertial force, and the load factor $\beta$ is as follows:

When the cylinder is moving slowly, $v<100 \mathrm{~mm} / \mathrm{s}, \beta=0.65$;

When the cylinder is moving, $v=100 \sim 500 \mathrm{~mm} / \mathrm{s}, \beta=0.5$;

When the cylinder moves at high speed, $v>500 \mathrm{~mm} / \mathrm{s}, \beta=0.35$.

The above formula is derived:

$$
D^{2}-d^{2}=\frac{4 \cdot F}{\pi \cdot p \cdot \beta}
$$

The smaller the air supply pressure $p$, the greater the $\left(D^{2}-d^{2}\right)$. The minimum air pressure of this smart pneumatic balance system is $0.6 \mathrm{MPa}$, and the air cylinder is medium speed.

This paper sets the load of intelligent pneumatic balance crane between $0 \sim 3000 \mathrm{~N}, \pi$ takes 3.14. The calculation formula is shown below.

$$
D^{2}-d^{2}=12738 \mathrm{~mm}^{2}
$$

In order to improve the efficiency of the balance crane, we take $D / d>5$. When $d<=15 \mathrm{~mm}, D>$ $111.9 \mathrm{~mm}$. So we choose $D=120 \mathrm{~mm}, d=15 \mathrm{~mm}$. 
According to the set workload, the selected cylinder, you can further select the regulator 2, solenoid valve 3 , solenoid valve 4 and other components.

Microcontroller Selection Analysis. MCU selection to a large extent determines the complexity of the hardware circuit. Highly integrated MCUs can reduce many peripheral devices. This paper chooses STC12C5A60S2 series microcontroller, it is high speed, low power consumption, strong anti-interference of a new generation of 8051 single chip microcomputer, high cost-effective, PCA/PWM, ISP (in system programmable) [8] [9].

Design of the Drive Circuit. The drive capability of the microcontroller cannot meet the needs of the relay. The design adopts ULN2003 as the driving device of the relay, and the TLP521-4 optcoupler isolation circuit is used to ensure the safety of the single chip. The circuit is shown in Figure 4.

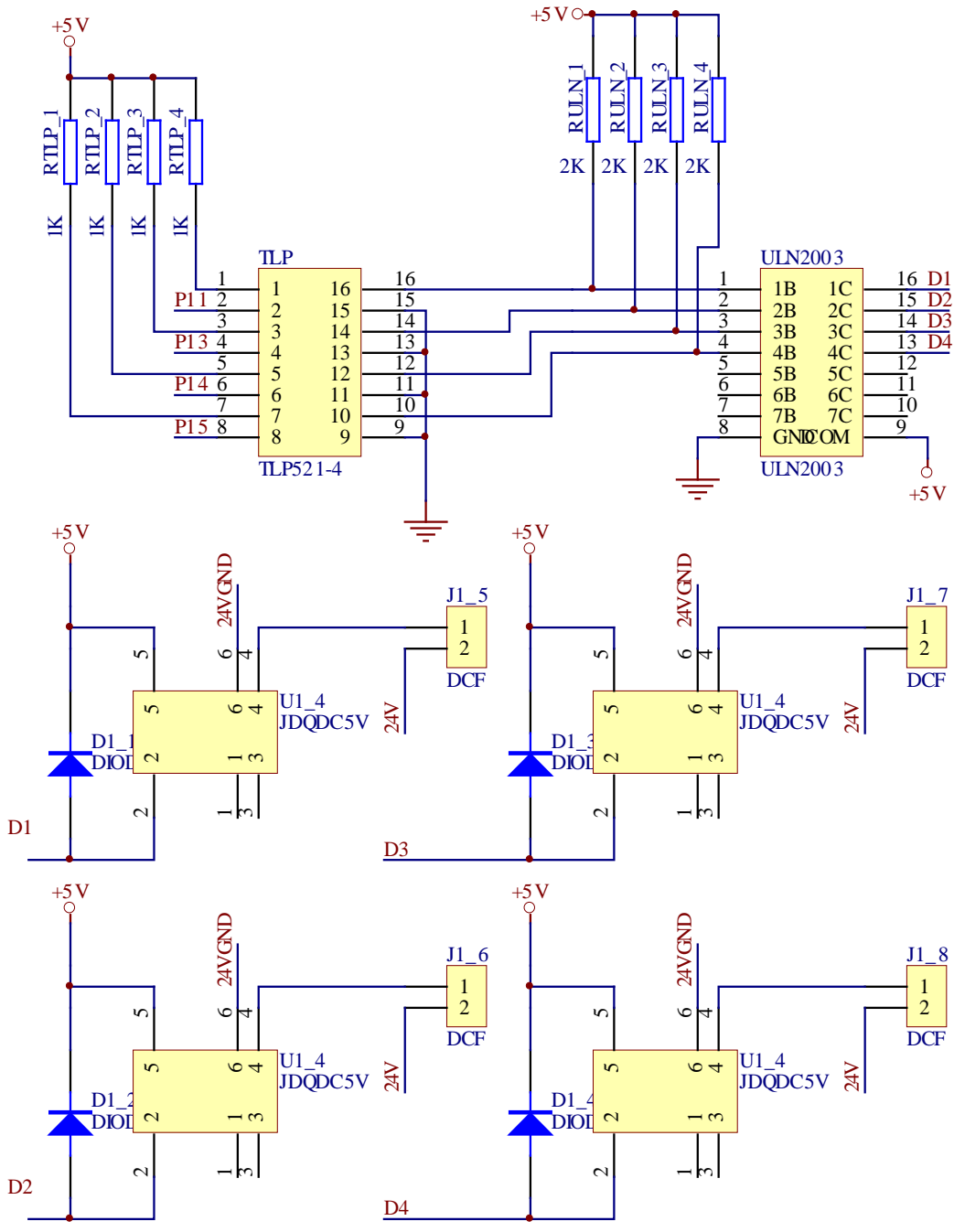

Fig.4. Drive circuit

The relay uses the environmental protection type of hk4100f-dc5v-shg model. Working voltage DC5V, sealed for the sealed package, power consumption of $0.2 \mathrm{~W}$.

ULN2003 is a high voltage, high current transistor array, composed of seven NPN composite transistors. When input 5VTTL level, the output up to $500 \mathrm{~mA} / 50 \mathrm{~V}$, it can directly drive the relay. This design requires only four of the seven transistors. Usually, when the single-chip microcomputer drives ULN2003, the pull-up 2K resistance is more appropriate.

The TLP521 optcoupler device completely isolates the microcontroller from the ULN2003-driven relays. It increases the safety of the controller and enhances the circuit's anti-jamming performance. 


\section{Design of the Software of Control System}

According to the system control requirements and functions, the program network map is drawn [10]. As shown in Figure 5.

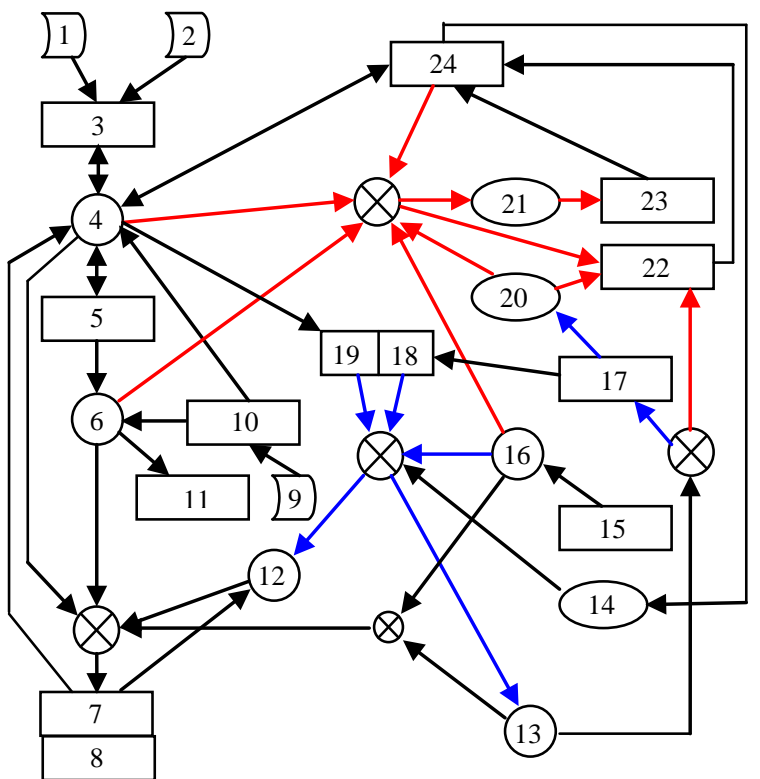

1-Plus or minus key. 2-FUN key. 3-Keystroke-scan. 4-Control signal. 5-Kd set. 6-Kd. 7-Adaptive ascent. 8-Adaptive descent. 9-PC. 10-The serial port receives interrupt. 11-ROM_write. 12-G_LF. $13-P$ p. $14-P d$ counter. $15-A D C$ interrupt routine.16-Pd. 17-Alarm program. 18-Decline. 19-rise. 20-Alarm information. 21-num[4]. 22-Serial port transmission. 23-Refresh LED. 24-T0 interrupt

Fig.5. Software design network diagram

The program first checks whether the balance system is in the operating mode or the parameter setting mode.

If it is a parameter model can be set parameters, and connected to the computer, using interrupt mode, on the computer software to set parameters and transmission, also can be set through the pressure display and setting circuit parameters.

If it is in the operating mode, call the initialization parameter, then wait for the key and detect the pressure to display. The main program flow chart is shown in Figure 7.

In the figure $|P d-P p|>K d, P d$ is the dynamic pressure value, $P p$ is the equilibrium pressure value after the rise and stop. For the adaptive pressure action threshold $K d$, for example, set to $3 \mathrm{Kg}$. When the weight is pressed down, the pressure of the compressed air $P d$ increases, and when the pressure generated by the pressure $P d$ is $3 \mathrm{Kg}$ greater than the equilibrium pressure $P d$, the Y2 moves exhaust and the weight moves downward. So, the smaller $K d$ is, the more sensitive the adaptation, but more prone to shock. The main program of the balance crane control system is shown in Figure 6 .

Td is to keep the threshold for a certain time and then action to prevent the shock caused by the system caused by false shocks to improve the stability of the system.

\section{Summary}

The use of micro-controller control and pneumatic system can make the ordinary cylinder to work as the pneumatic balance crane, which may simplify the structure of the commonly used balance crane, shorten the product manufacturing cycle. It also creases the safety of the use of the product because of using self-control safety valve airway protection.

The prototype test shows that the new smart pneumatic balance crane is simple and easy to operate, and is suitable for most mechanical assembly, sheet metal forming, machine tool and other industries. The prototype is shown in Figure 7. 


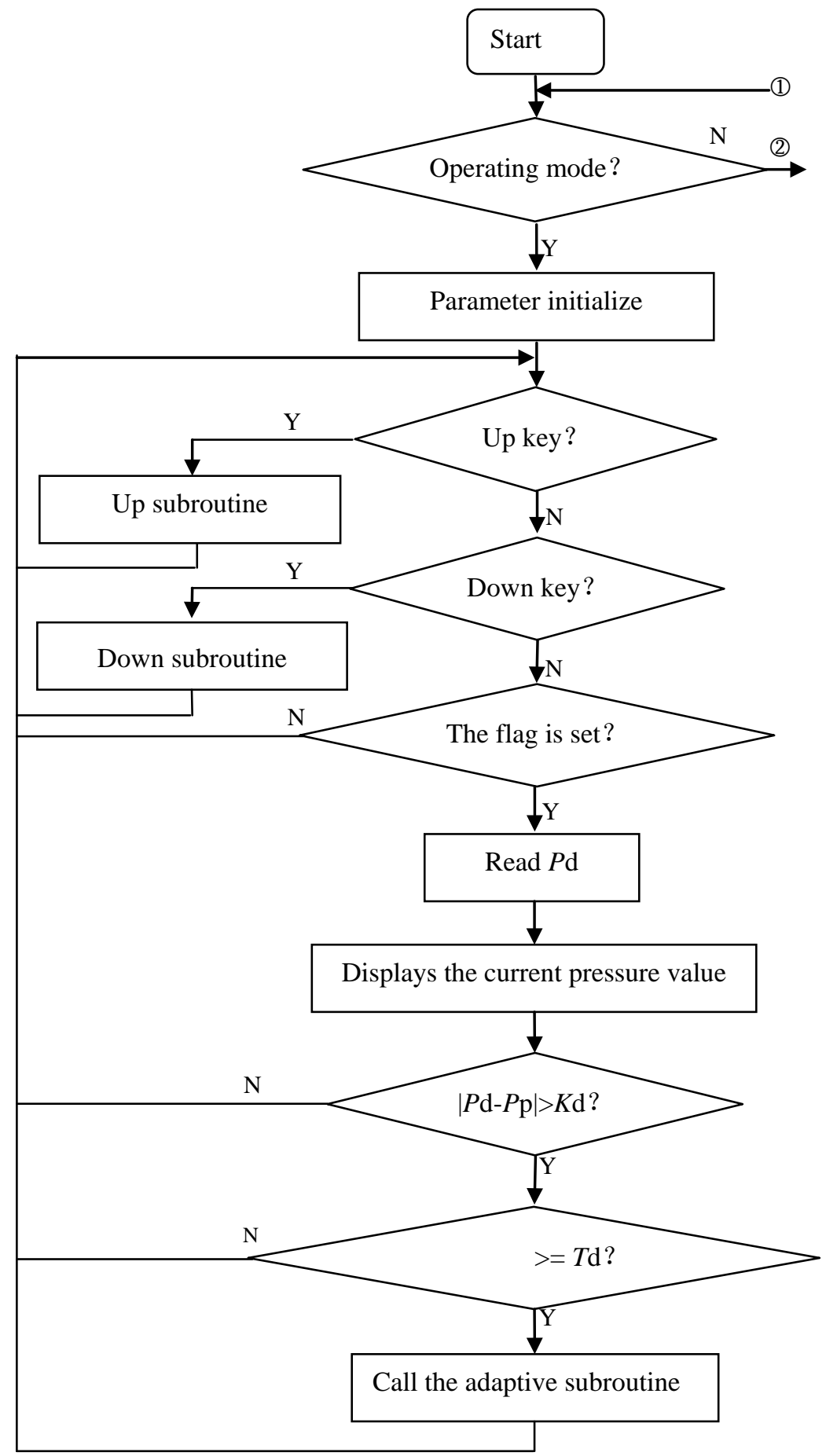

(1)

(2)

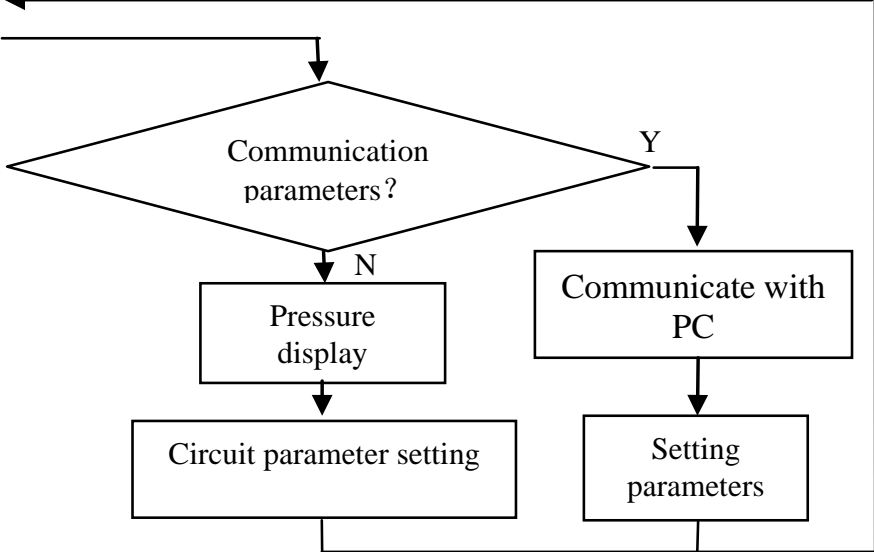


Fig.6. Main program of the balance crane control system

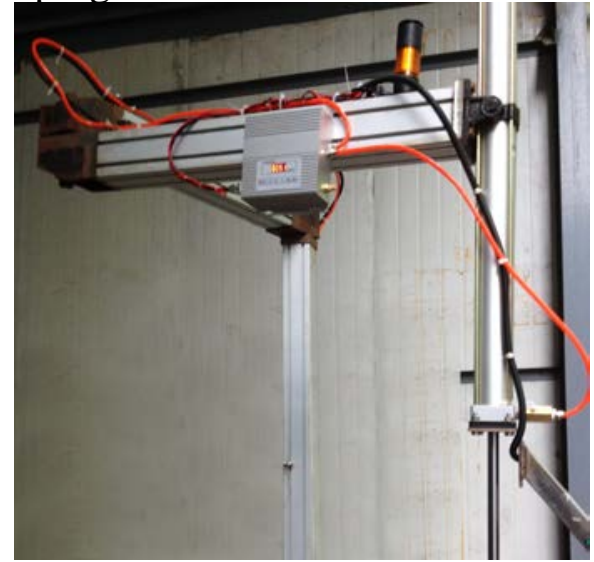

Fig.7. Application engineering site

\section{Acknowledgements}

This work is supported by The Research Project of Higher Vocational Education in 2017 of Nanjing Vocational Institute of Industry Technology (GJ17-04Z); supported by High-end Training Funded Projects of Jiangsu Vocational Institute Professional Leader (2016GRFX032); and also sponsored by Jiangsu university excellent science and technology innovation team funding project (2015SJTD-01)

\section{References}

[1] Hongxing Li, Qin Sun. Application of Pneumatic Balance System in Product Assemble Line[J].Chinese Hydraulics \& Pneumatics, 2013(1):43-44.

[2] Sujie Xu. Development and Application of a Kind of Pneumatic gourd[J]. Machinery manufacturing and automation, 2012(8): 59-60.

[3] Shunqiang Shang. Research of Hydraulic Balance Crane with Manifold Block[J]. Chinese Hydraulics \& Pneumatics,2012(6):51-53.

[4] Jiujun Zhen, Zhanmin Yang, Xiaoyong Wang, et al. An adaptive pressure balance control system based on microcontroller: China, 201520289813.7[P].2015-08-26.

[5] Xiuli Qi, Liang Liu, Bin Ju. Design of Controlled Loop for Adaptive Pneumatic Lifting Equipment[J]. Machine tools and hydraulic, 2015, 43(10): 96-97.

[6] Lijun Xiao, Xuening Mi, Lei Shi, et al. Principle and Application of Power Balance Pneumatic Manipulator[J]. Chinese Journal of Manufacturing Automation, 2011, 33 (6):230-232.

[7] Chong Yang, Kun Chen. Design and application of the pneumatic assisted Balance Crane [J].Chinese Journal of Machine China, 2013(2):103-104.

[8] Feng Wang, Ruan Zhao. Design of Autonomous Dredging Device Control System Based on AT89S52[J]. Machine tools and hydraulic, 2013, 40(20): 121-123.

[9] Chao Liu, Xinmei Liu, Wen Hou. An Automatic Balance Sling with Microprocessor Control[J]. Chinese Journal of Shanxi Electronic Technology, 2012 (2):22-23.

[10] Liyana Ramli, Z. Mohamed, Auwalu M. Abdullahi, H.I. Jaafar, Izzuddin M. Lazim. Control strategies for crane systems: A comprehensive review [J]. Mechanical Systems and Signal Processing, Volume 95, October 2017, Pages 1-23.000 